IInstitute for Global Health, University College London. Londres, Reino Unido. Escuela de Enfermería, Universidad de los Andes. Santiago, Chile. ${ }^{3}$ Escuela de Ingeniería, Pontificia Universidad Católica de Chile. Santiago, Chile. ${ }^{4}$ Departamento Salud Pública, Escuela de Medicina, Pontificia Universidad Católica de Chile. Santiago, Chile.

${ }^{5}$ Advanced Center for Chronic Diseases (ACCDiS), FONDAP, Chile

${ }^{6}$ Centro de Desarrollo Urbano Sustentable (Cedeus), FONDAP, Chile

${ }^{7}$ Departamento de Medioambiente, Aseo y Ornato, llustre Municipalidad de Renca, Chile.

${ }^{8}$ Escuela de Salud Pública, Universidad de Chile. Santiago, Chile aEnfermera, Magíster en Salud Pública, Ph.D. ${ }^{\circ}$ Global Health.

bIngeniero civil, Master in Civil Engineering,

Ph.D. Engineering and Public Policy.

'Médico veterinario, Magíster en Ciencias

Biológicas, Doctor en Salud Pública.

dIngeniero Ambiental, Magíster en Gobierno y Gerencia Pública.

ePsicólogo, Master Global Health and Development.

Trabajo no recibio financiamiento Los autores declaran no tener conflictos de interés.

Recibido el 31 de diciembre de 2019, aceptado el 23 de julio de 2020.

Correspondencia a: Yasna K. Palmeiro-Silva

Dirección: Flat 5, Jerome House, 5-13 Glendower Place, Londres, Reino Unido. SW7 3DU. yasna.palmeiro.18@ucl.ac.uk

\section{La amenaza del cambio climático a la salud de la población y la necesidad urgente de actuar}

\author{
YASNA K. PALMEIRO-SILVA ${ }^{1,2, a,}$, LUIS A. CIFUENTES ${ }^{3, \mathrm{~b}}$, \\ SANDRA CORTÉS ${ }^{4,5,6, c}$, MACARENA OLIVARES ${ }^{7, d}$, \\ IGNACIO SILVA $8, \mathrm{e}$
}

\section{The threat of climate change on population health and the urgent need to act}

Climate change is associated with negative health outcomes, such as cardiovascular and respiratory diseases. This article analyses the threat of climate change on population health and the urgent need to implement measures to avoid this damage. Heat vulnerability, heatwave exposures, and wildfire exposure to forest fires have increased in Chile. In 2018, the annual mean concentration of fine particulate matter $\left(P M_{2.5}\right)$ exceeded the safe level proposed by the World Health Organization, increasing the risk of negative health outcomes. Thus, multidisciplinary and intersectoral mitigation and adaptation policies are needed. Among other elements, mental health impacts of climate change, health education provided by health workers to reduce negative health impacts of climate change, greater engagement of academia to generate evidence useful for policy-making processes and a better articulation between central and local governments should be considered. Finally, achieving a healthy population should be the aim of all these policies and efforts.

(Rev Med Chile 2020; 148: 1652-1658)

Key words: Climate Change; Environment and Public Health; Population Health; Public Policy.
$\mathrm{D}$ urante miles de años, el ser humano ha utilizado recursos provistos por el medioambiente y al mismo tiempo lo ha modificado. El mayor ejemplo de esto es la revolución industrial, que por una parte favoreció el crecimiento industrial y tecnológico, lo que impulsó el bienestar de la población, la disponibilidad de alimentos, los modos de transporte, entre otros ${ }^{1}$; pero por otra parte, el uso de carbón y otros combustibles fósiles como fuente de energía, incrementó la polución ambiental y emisiones de dióxido de carbono, y con ello problemas de salud en la población ${ }^{2,3}$. Estas consecuencias negativas eran insospechadas en ese entonces, ya que las enfermedades infecciosas y carenciales, la alta mortalidad materno-infantil, el desarrollo poblacional y el crecimiento económico eran temas más importantes en términos de política y administración pública, por lo tanto, las potenciales consecuencias derivadas de actividades humanas sobre el medioambiente no fueron consideradas como un problema público de salud.

El continuo uso de combustibles fósiles ha liberado toneladas de gases de efecto invernadero (GEI) a la atmosfera, potenciando el efecto invernadero natural de la tierra. En palabas simples, 
el aumento de la concentración de GEI en la atmósfera conlleva a que esta atrape más calor, lo que resulta en un planeta más caliente, fenómeno conocido como calentamiento global. Esto se ha reflejado en un aumento de $0,85^{\circ} \mathrm{C}$ de la temperatura de la superficie terrestre y oceánica entre 1880 y 2012 en comparación con niveles preindustriales ${ }^{4}$. Este aumento de la temperatura global afecta el sistema climático de la tierra, provocando cambios en el clima o cambio climático, definido como cambios estadísticamente significativos en la media y/o variabilidad del clima, típicamente, sobre 30 años y que podrían ser provocados por forzantes internos o externos al sistema, incluyendo la actividad humana ${ }^{4,5}$.

Los efectos del cambio climático se pueden observar en todo el mundo, pero los impactos difieren debido a características y vulnerabilidades regionales y locales. En particular, el cambio climático está afectando a las poblaciones humanas y exacerbando inequidades sociales y problemas de salud existentes ${ }^{6,7}$. En general, los efectos en salud se relacionan a calor extremo, contaminación del aire, disponibilidad de agua y comida, eventos climáticos extremos, entre otros, y sus impactos se observan en términos de morbimortalidad ${ }^{8,9}$. Consecuentemente, esta situación pone en jaque a los sistemas de salud, los que deben prepararse y responder activamente ante las crecientes necesidades de la población y así proveer una atención en salud adecuada ${ }^{10}$.

El propósito de este artículo es analizar la amenaza del cambio climático sobre la salud de la población y la necesidad urgente de implementar medidas de mitigación y adaptación integrales, eficientes, multidisciplinarias e intersectoriales, con el objetivo de proteger la salud de todas las personas, especialmente las más vulnerables. Este documento incluye evidencia científica recogida en la literatura, pero también se basa en las discusiones sostenidas en el seminario "Efectos del cambio climático en la salud: Presentación del reporte para Chile de Lancet Countdown 2019"11.

\section{Cambio climático y salud: Escenarios internacional y nacional}

En términos simples, el cambio climático puede generar diferentes impactos en la salud de la población a través de efectos directos e indirectos.
En la Figura 1 se observa que los efectos directos, como olas de calor y calor extremo, pueden finalizar en eventos de mayor morbimortalidad o alteración del bienestar de la población debido a estrés por calor, pero estos impactos están mediados por diferentes determinantes sociales de la salud, los que podrían aumentar o disminuir la magnitud del resultado final. Paralelamente, el cambio climático altera elementos del ecosistema, por ejemplo, provocando inundaciones o sequias, lo que perturba los sistemas humanos, como la disponibilidad de comida o agua. Esto cambios impactan la salud de la población, todo esto nuevamente mediado por los determinantes sociales de la salud.

Con el objetivo de evaluar el perfil de salud del cambio climático, la colaboración internacional The Lancet Countdown ha analizado diversos indicadores de salud relacionados al cambio climático. Se desprende del reporte 2019, que más del 77\% de los países han experimentado un incremento en la exposición diaria de la población a incendios forestales en el período 2015-2018 en comparación al 2001-2004. La exposición de adultos mayores a olas de calor ha aumentado en el mundo, lo que conlleva mayor riesgo de eventos negativos en salud. Finalmente, el número de muertes asociadas a material particulado fino $\left(\mathrm{PM}_{2.5}\right)$ se ha mantenido constante en 2,9 millones de personas en 2016, demostrando mínimos avances en esta problemática a nivel mundial y lo que podría ser aún peor si se considerara el impacto del carbón negro (black carbon) sobre la salud de las personas ${ }^{12}$. Considerando estas cifras, el reporte plantea que en el caso de continuar con el mismo ritmo de emisiones de GEI, la salud de las personas estará cada vez más determinada por el cambio climático $^{13}$. Sin embargo, existe una vía que aboga por limitar el incremento de la temperatura global a no más allá de $2^{\circ} \mathrm{C}$ e incluso hasta $1.5^{\circ} \mathrm{C}$, según lo establecido en el Acuerdo de Paris ${ }^{14}$. El reporte plantea que bajo este escenario, los niños y niñas nacidas hoy en día podrían vivir en un mundo más seguro, limpio y donde su salud no dependa del cambio climático ${ }^{13}$.

La población chilena es vulnerable a los efectos del cambio climático por varias razones. Primero, parte importante del crecimiento económico del país ha sido basado en actividades altamente sensibles al cambio climático, como lo son la agricultura, fruticultura, vinicultura, silvicultura 


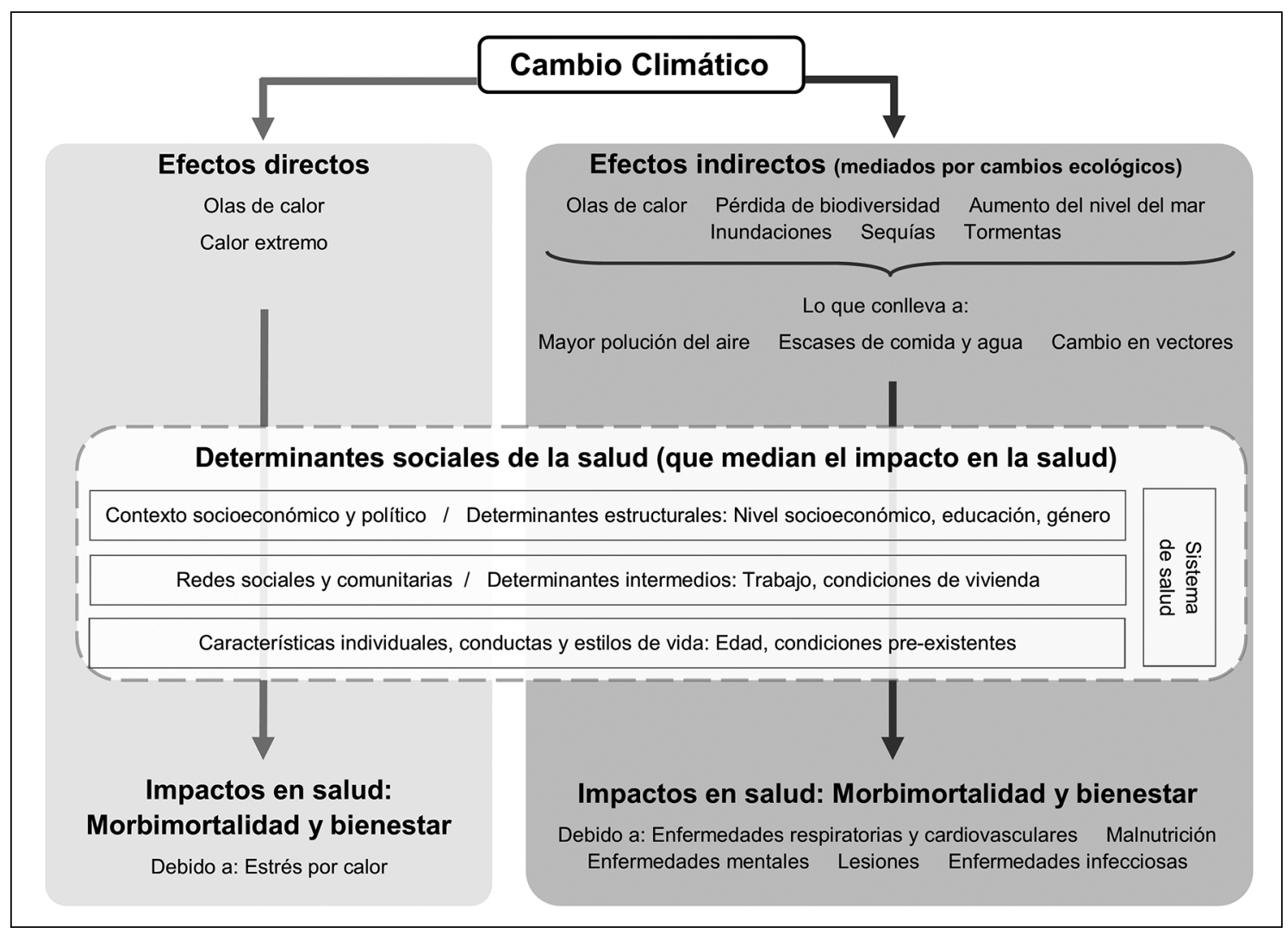

Figura 1. Vías por la que el cambio climático afecta la salud de la población.

y acuicultura. Segundo, una parte importante de la población del país es vulnerable socialmente ${ }^{15}$, situación que la pone en mayor riesgo frente a los impactos del cambio climático. Tercero, la proporción de adultos mayores de 65 años y personas con enfermedades no transmisibles ha aumentado en el país ${ }^{16,17}$, lo que aumenta la probabilidad de experimentar eventos negativos en salud asociados al cambio climático. Cuarto, Chile presenta 7 de 9 características que la Convención Marco de Naciones Unidas sobre el Cambio Climático ha destacado como de especial preocupación: (i) zonas costeras bajas, (ii) áreas áridas, semiáridas o boscosas, (iii) áreas propensas a desastres debido a peligros naturales, (iv) áreas sujetas a sequía y desertificación, (v) áreas urbanas con alta contaminación atmosférica, (vi) áreas con ecosistemas frágiles, (vii) economías dependientes de productos intensivos en energía ${ }^{5}$. Quinto, el país presenta una gobernanza e institucionalidad medioambien- tal relativamente joven, lo que podría ser visto como una oportunidad o amenaza, según como se desarrollen las políticas ambientales.

En términos de salud y cambio climático en Chile, existe escasa evidencia disponible. En 2008, un estudio estimó que temperaturas elevadas se asocian a un mayor riesgo de mortalidad en personas mayores de 65 años en ciudades de Latinoamérica $^{18}$. Complementariamente, Cifuentes et al., concluye que de acuerdo con las alzas de temperatura proyectadas para Chile, la tasas de ingresos hospitalarios podrían aumentar hacia el año $2099^{19}$. En 2016, se lanza el Plan de Adaptación al Cambio Climático del sector Salud, el cuál plantea ocho ejes de acción ${ }^{20}$, pero que la mayoría de ellos necesita como primera instancia un diagnóstico de la situación actual de los impactos del cambio climático en la salud de la población, los que hasta la fecha no se han evaluado completamente. 
The Lancet Countdown ha analizado información respecto a la situación del cambio climático y salud en Chile. En particular, el resumen de políticas para Chile ha destacado tres problemáticas: olas de calor, incendios forestales y contaminación del aire ${ }^{21}$. Este documento enfatiza que el índice de vulnerabilidad al calor ha aumentado en 7,7\% desde 1990, al igual que el número de exposiciones a olas de calor por parte de adultos mayores. La exposición a incendios forestales se ha casi triplicado al comparar los períodos 2001-2004 y 2015-2018, aumentando el riesgo de ocurrencia de eventos negativos en salud, tanto directos como indirectos. Finalmente, el informe destaca que la concentración promedio anual de $\mathrm{PM}_{2.5}$ a nivel nacional alcanzó $24,9 \mu \mathrm{g} / \mathrm{m}^{3}$ en 2018 , lo que sobrepasa el nivel recomendado por la Organización Mundial de la Salud $\left(10 \mu \mathrm{g} / \mathrm{m}^{3}\right)$ y la norma primaria de calidad del aire de Chile $\left(20 \mu \mathrm{g} / \mathrm{m}^{3}\right)$, siendo este nivel mucho más elevado en ciertas ciudades y comunas del país como Padre las Casas $(43,3 \mu \mathrm{g} /$ $\left.\mathrm{m}^{3}\right)$, Coyhaique $\left(34,2 \mu \mathrm{g} / \mathrm{m}^{3}\right)$ y Osorno $(38,2 \mu \mathrm{g} /$ $\left.\mathrm{m}^{3}\right)^{21}$. Cabe destacar que estos datos han sido obtenidos desde el reporte 2018 World Air Quality 22 , por lo que difieren de los valores informados en el Sistema de Información Nacional de Calidad del Aire (SINCA), los cuales arrojan cifras mayores debido a una posible diferencia en la estimación. Palmeiro et al. destacan que durante el año 2016 en Chile, 2.830 muertes prematuras se asociaron a exposición a combustibles para uso residencial, 620 a transporte terrestre, 500 a contaminación por procesos industriales y 80 debido al uso de carbón en plantas de energía e industria ${ }^{13}$.

\section{Soluciones: Una mirada multidisciplinaria e intersectorial}

Ante la situación anteriormente descrita, es necesario plantear políticas de mitigación y adaptación al cambio climático con una mirada multidisciplinaria e intersectorial y que aboguen por la protección de la salud de la población. Si bien el informe de resumen de políticas para Chile publicado por The Lancet Countdown propone recomendaciones para la política pública, estas deben ser analizadas por actores sociales de diversas disciplinas e incluyendo sectores como energía, industria, salud, gobiernos locales, academia y la ciudadanía.
Como una primera aproximación, el pasado 3 de diciembre se realizó un seminario que puso sobre la mesa esta problemática y que incluyó diversas miradas ${ }^{11}$. Algunos puntos destacados de la discusión fueron los siguientes:

\section{Cambio climático y salud mental}

Los efectos del cambio climático, especialmente los eventos meteorológicos extremos e incendios, afectan la salud mental de niños, niñas y adolescentes, destacando el estrés postraumático, ansiedad y depresión ${ }^{23-25}$, lo que a su vez impacta en la regulación emocional, cognición y desarrolo escolar. Ante esto, es necesaria una respuesta efectiva por parte de los sistemas de salud proporcionando un sistema de protección social a los niños, niñas y adolescentes más vulnerables, que aborde integralmente las diferencias sociales que podrían aumentar su vulnerabilidad. Además, los medios de comunicación tienen responsabilidad de informar adecuadamente y comunicar los riesgos y potenciales soluciones.

\section{Rol de los profesionales de la salud}

Los trabajadores de la salud son uno de los grupos más confiables en la sociedad ${ }^{26}$, por lo tanto deben cumplir un rol activo en la temática de cambio climático y salud, promoviendo medidas de adaptación a distintos niveles, tanto individual como comunitario y central. Los trabajadores de la salud deben educar a la población en promoción y prevención, incluyendo recomendaciones que permitan reducir los impactos del cambio climático. Complementariamente, es necesario que se involucren en la toma de decisión para impulsar acciones que protejan la salud pública como por ejemplo, sistemas de vigilancia, preparación para desastres o control de enfermedades ${ }^{27}$.

\section{Rol de la academia}

La academia debe involucrarse mucho más en la formulación de políticas públicas y producción de evidencia científica de alta calidad que informe adecuadamente los procesos de toma de decisión a distintos niveles. Complementariamente, es necesario que el gobierno, políticos y tomadores de decisión trabajen con académicos para la formulación de políticas públicas ${ }^{28}$, de esta manera, el know-how de la academia como el de la política pública se unen y potencian para el desarrollo de una política en salud ambiental basada en eviden- 
cia. Paralelamente, las instituciones de educación, de diferentes niveles y disciplinas, deben incorporar la temática del cambio climático y salud en las mallas curriculares y con ello formar ciudadanos conscientes del problema y comprometidos con las soluciones.

\section{Gobiernos locales}

Los gobiernos locales son de gran relevancia para la ejecución de acciones de mitigación y adaptación, por ello debe existir una clara articulación entre la política nacional y las capacidades de los gobiernos locales, ya que de esta manera será posible realizar diagnósticos comunitarios y planes locales de acción que se podrán implementar y evaluar de manera mucho más eficiente ${ }^{29}$.

Considerando la discusión del seminario anteriormente señalado y desde un punto de vista más general, es necesario revisar las políticas públicas de mitigación y adaptación de nuestro país en relación con la salud poblacional. El Plan Nacional de Adaptación de Cambio Climático 2017-2022 plantea 96 medidas y solo una de ellas hace referencia a salud ${ }^{30}$, sin mencionarse explícitamente la necesidad de proteger el bienestar y la salud de la población. Por otra parte, al revisar los planes de adaptación sectoriales disponibles (sector silvoagropecuario, pesca y acuicultura, biodiversidad, servicios de infraestructura y ciudades) se observa escasa mención a la relación de cambio climático y salud, y la necesidad de mitigar o adaptarse para proteger la salud de la población ${ }^{31-35}$, a pesar de que impactos climáticos a nivel de agricultura, infraestructura o biodiversidad afectan el bienestar y salud humana. Finalmente, la Contribución Nacional Determinada presentada por Chile el año 2015 incluye elementos en mitigación y adaptación al cambio climático, aunque algunas medidas son generales y condicionales a financiación externa. Por otra parte, no se incluyen medidas específicas que aboguen por la protección del bienestar y salud de la población ${ }^{36}$.

Si bien se entiende que las políticas públicas deben ser factibles de llevar a cabo, es necesario que la salud esté presente en todas las políticas y no solo en la política de salud. La aproximación de salud en todas las políticas se basa en el principio del co-beneficio y ha sido definida como "un enfoque de las políticas públicas en todos los sectores, que sistemáticamente toma en cuenta las implicaciones de las decisiones sobre la salud, busca sinergias y evita los efectos nocivos sobre la salud, con el propósito de mejorar la salud de la población y la equidad en salud"37, promoviendo la colaboración entre diferentes actores de gobierno, organizaciones comunitarias, empresas, entre otros. De esta manera, el sistema pone a las personas, familias y comunidades en el centro de la política, considerando el bienestar y un buen nivel de salud como objetivo común y final, pero que a su vez reconoce que una población saludable trae varios beneficios económicos y sociales ${ }^{38}$.

Como conclusión, el llamado es hacia una mayor ambición climática por parte del gobierno, instituciones públicas y privadas, comunidades e individuos para tomar acciones en mitigación y adaptación al cambio climático, y a través de ellas, proteger a las personas y el medioambiente, contribuyendo a una mayor justicia social. De igual manera, el sistema de salud, incluyendo instituciones y personas, debe ser una voz clara y actor clave en la generación y articulación de la política pública en este ámbito.

Agradecimientos: YKPS agradece a 1) CONICYT Programa de Formación de Capital Humano Avanzado Becas de Doctorado en el Extranjero Becas Chile 2018 - 72190338; 2) Institute for Global Health de la University College London; 3) Colaboración The Lancet Countdown; 4) Centro de Políticas Públicas UC.

\section{Referencias}

1. Allen RC. The British Industrial Revolution in Global Perspective (New Approaches to Economic and Social History). Cambridge, United Kingdom: Cambridge University Press; 2009.

2. White M. The Industrial Revolution. The British Library; 2009. Disponible en: https://www.bl.uk/georgian-britain/articles/the-industrial-revolution [Consultado el 11 de marzo de 2019].

3. Bailey RE, Hatton TJ, Inwood K. Atmospheric Pollution and Child Health in Late Nineteenth Century Britain. IZA DP No. 10428. 2016.

4. Intergovernmental Panel on Climate Change. Climate Change 2013: The Physical Science Basis. Working Group I Contribution to the Fifth Assessment Report of the Intergovernmental Panel on Climate Change. Stocker T, Qin D, Plattner G-K, Tignor MMB, Allen SK, 
Boschung J, et al., editors. Cambridge, United Kingdom and New York, NY, USA.: Cambridge University Press; 2013.

5. United Nations. United Nations Framework Convention on Climate Change. 1992.

6. Watts N, Adger WN, Agnolucci P, Blackstock J, Byass P, Cai W, et al. Health and climate change: policy responses to protect public health. Lancet. 2015; 386 (10006): 1861-914.

7. Intergovernmental Panel on Climate Change. Climate Change 2014: Impacts, Adaptation, and Vulnerability. Part A: Global and Sectoral Aspects. Working Group II Contribution to the Fifth Assessment Report of the Intergovernmental Panel on Climate Change. In: Arros VR, Field CB, Jon Dokken D, Mastrandrea MD, Mach KJ, Bilir TE, et al., editors. Cambridge, United Kingdom and New York, NY, USA.: Cambridge University Press; 2014.

8. Smith KR, Woodward A, Campbell-Lendrum D, Chadee DD, Honda Y, Liu Q, et al. Human Health: Impacts, Adaptation, and Co-Benefits. In: Field CB, Barros VR, Jon Dokken D, Mach KJ, Mastrandrea MD, Bilir TE, et al., editors. Climate Change 2014: Impacts, Adaptation, and Vulnerability Part A: Global and Sectoral Aspects Contribution of Working Group II to the Fifth Assessment Report of the Intergovernmental Panel on Climate Change. United Kingdom and New York, NY, USA: Cambridge University Press; 2014. p. 709-54.

9. McMichael AJ, Woodruff RE, Hales S. Climate change and human health: present and future risks. Lancet. 2006; 367 (9513): 859-69.

10. Frumkin H, Hess J, Luber G, Malilay J, McGeehin M. Climate change: the public health response. Am J Public Health 2008; 98 (3): 435-45.

11. Centro de Políticas Públicas UC. Seminario Efectos del cambio climático en la salud: Presentación del reporte para Chile de Lancet Countdown 2019. Disponible en: https://politicaspublicas.uc.cl/seminario/seminario-efectos-del-cambio-climatico-en-la-salud-presentacion-del-reporte-para-chile-de-lancet-countdown-2019/ [Consultado el 11 de diciembre de 2019].

12. Janssen NAH, Hoek G, Simic-Lawson M, Fischer P, van Bree L, Brink $\mathrm{H}$ Ten, et al. Black carbon as an additional indicator of the adverse health effects of airborne particles compared with PM10 and PM2.5. Environ Health Perspect 2011; 119 (12): 1691-9.

13. Watts N, Amann M, Arnell N, Ayeb-Karlsson S, Belesova K, Boykoff M, et al. The 2019 report of The Lancet Countdown on health and climate change: ensuring that the health of a child born today is not defined by a changing climate. Lancet 2019; 394 (10211): 1836-78.
14. United Nations Framework Convention on Climate Change. Paris Agreement. 2015.

15. Ministerio de Desarrollo Social y Familia (CHL). Observatorio Social: Encuesta CASEN: base de datos. Santiago de Chile. 2017. Disponible en: http://observatorio.ministeriodesarrollosocial.gob.cl/casen-multidimensional/ casen/casen_2017.php [Consultado el 12 de diciembre de 2019].

16. Instituto Nacional de Estadisticas. Estimaciones y Proyecciones de la Población de Chile 1992-2050 (Total País) 2017. Disponible en: https://www.censo2017.cl/ [Consultado el 12 de diciembre de 2019].

17. Ministerio de Salud (CHL). Encuesta Nacional de Salud 2016-2017 Primeros resultados. 2017. Disponible en: https://www.minsal.cl/wp-content/uploads/2017/11/ ENS-2016-17_PRIMEROS-RESULTADOS.pdf [Consultado el 12 de diciembre de 2019].

18. Bell ML, O’Neill MS, Ranjit N, Borja-Aburto VH, Cifuentes LA, Gouveia NC. Vulnerability to heat-related mortality in Latin America: a case-crossover study in São Paulo, Brazil, Santiago, Chile and Mexico City, Mexico. Int J Epidemiol 2008; 37 (4): 796-804.

19. GreenLabUC. Identificación de impactos, evaluación de vulnerabilidad del sector salud frente al cambio climático y propuestas para la adaptación. Santiago, Chile. 2012. Disponible en: https://www.greenlab.uc.cl/ wp-content/uploads/2013/11/2012-IISCC-InformeFinal. pdf [Consultado el 12 de diciembre de 2019].

20. Ministerio de Salud, Ministerio del Medio Ambiente (CHL). Plan de Adaptación al Cambio Climático Sector Salud. 2017. Disponible en: https://mma.gob.cl/wp-content/uploads/2018/06/06_04Plan-CC-SALUD-2016.pdf [Consultado el 12 de diciembre de 2019].

21. Palmeiro-Silva YK, Cifuentes LA, Cortés S. The Lancet Countdown Policy Brief for Chile. 2019.

22. IQ Air Air Visual. 2018 World Air Quality Report. 2019.

23. Morrissey SA, Reser JP. Natural disasters, climate change and mental health considerations for rural Australia. Aust J Rural Health 2007; 15 (2): 120-5.

24. Doherty TJ, Clayton S. The Psychological Impacts of Global Climate Change. Am Psychol 2011; 66 (4): 26576.

25. Hayes K, Blashki G, Wiseman J, Burke S, Reifels L. Climate change and mental health: risks, impacts and priority actions. Int J Ment Health Syst. 2018;12(1):28.

26. Ipsos. Ipsos MORI Veracity Index 2019: Trust in professions survey. 2019.

27. Kreslake JM, Sarfaty M, Roser-Renouf C, Leiserowitz AA, Maibach EW. The Critical Roles of Health Professionals in Climate Change Prevention and Preparedness. Am J Public Health 2018; 108 (S2): S68-9. 
28. Sasse T, Haddon C. How academia can work with government. 2019.

29. Department for Environment Food \& Rural Affairs, ADEPT. Preparing for a changing climate: Good practice guidance for local government. 2019.

30. Ministerio del Medio Ambiente (CHL). Plan de Acción Nacional de Cambio Climático 2017-2022. 2017. Disponible en: https://mma.gob.cl/wp-content/ uploads/2017/07/plan_nacional_climatico_2017_2.pdf [Consultado el 12 de diciembre de 2019].

31. Ministerio de Agricultura, Ministerio del Medio Ambiente (CHL). Plan de adaptación al cambio climático del sector silvoagropecuario. 2013. Disponible en: https://mma.gob.cl/wp-content/uploads/2015/02/ Plan_Adaptacion_CC_S_Silvoagropecuario.pdf [Consultado el 12 de diciembre de 2019].

32. Ministerio de Economia, Fomento y Turismo, Subsecretaria de Pesca y Acuicultura, Ministerio del Medio Ambiente (CHL). Plan de adaptación al cambio climático para pesca y acuicultura. 2016. Disponible en: https://mma.gob.cl/wp-content/uploads/2016/12/ Plan-Pesca-y-Acuicultura-CMS.pdf [Consultado el 12 de diciembre de 2019].

33. Ministerio del Medio Ambiente (CHL). Plan de adaptación al cambio climático en biodiversidad. 2014. Dis- ponible en: http://metadatos.mma.gob.cl/sinia/PDF008. pdf [Consultado el 12 de diciembre de 2019].

34. Ministerio de Obras Publicas, Ministerio del Medio Ambiente (CHL). Plan de adaptación y mitigación de los servicios de infraestructura al cambio climático 2017-2022. 2017. Disponible en: https://mma.gob.cl/ wp-content/uploads/2017/12/Plan-de-Accion-MOP.pdf [Consultado el 12 de diciembre de 2019].

35. Ministerio del Medio Ambiente (CHL). Plan de adaptación al cambio climático para ciudades 2018-2022. 2018. Disponible en: https://mma.gob.cl/wp-content/ uploads/2018/06/Plan-CC-para-Ciudades_aprobado-CMS-ene2018-1.pdf [Consultado el 12 de diciembre de 2019].

36. Gobierno de Chile (CHL). Intended Nationally Determined Contribution of Chile Towards the Climate Agreement of Paris 2015. 2015. Disponible en: https:// www4.unfccc.int/sites/submissions/INDC/Published\%20 Documents/Chile/1/INDC\%20Chile\%20english\%20 version.pdf [Consultado el 12 de diciembre de 2019].

37. World Health Organization, Finland Ministry of Social Affairs and Health. Health in all policies: Helsinki statement. Framework for country action. 2014.

38. Public Health England, Local Government Association. Local wellbeing, local growth: overview. 2016. 